\title{
Cambios temporales en la abundancia y composición del ensamble de aves acuáticas en tranques artificiales en Chile central
}

\section{Seasonal changes in the abundance and composition of the waterbirds assemblage in artificial ponds of Central Chile}

\author{
Isaac Peña-Villalobos, Pablo Fibla, Juan-Esteban Salazar \& Michel Sallaberry A*. \\ Laboratorio de Zoología de Vertebrados, Departamento de Ciencias Ecológicas, \\ Facultad de Ciencias, Universidad de Chile, Chile \\ *E-mail: msallabe@uchile.cl
}

\section{RESUMEN}

La zona central de Chile se caracteriza por ser un ambiente de tipo mediterráneo con marcada estacionalidad climática. En esta zona de gran actividad agrícola existen tranques artificiales destinados al regadío, los cuales pueden generar ambientes que constituyen hábitats alternativos para el desarrollo y nidificación de aves acuáticas. A pesar de la existencia de datos censales en este tipo de tranques, no se han realizado trabajos que aborden la estacionalidad climática como un factor implicado en la variación temporal de la abundancia y del ensamble ornitológico. En este trabajo, se estudiaron los cambios temporales de las comunidades de aves en dos tipos de tranques en la Región Metropolitana (33 $29^{\prime} \mathrm{S}-70^{\circ} 54^{\prime} \mathrm{W}$ ): un tranque de tipo estacional con régimen pluvial, que se seca durante la época estival y otro que se alimenta en forma controlada de aguas servidas. Sólo en el ambiente de régimen pluvial se observó variación estacional de la abundancia, la que se correlacionó positivamente con el índice de aridez mensual de Martonne. Por otra parte, en el ambiente de régimen controlado se registró sólo una tendencia en el aumento de la abundancia en el periodo estival, sin un incremento significativo. Además, se observaron en los dos tipos de tranques cambios estacionales en la composición de los ensambles, variación numérica de ciertas poblaciones y dispersión de aves entre tranques cercanos por efectos de desecación estival. Finalmente, se discute la importancia de los tranques de régimen controlado (e.g. plantas de tratamiento o tranques de aguas servidas) como hábitats estivales opcionales durante el proceso de desecación de sitios estacionales.

Palabras Clave: Aves acuáticas, estacionalidad, índice de aridez de Martonne, humedales, tranques.

\begin{abstract}
Central Chile is characterized by a Mediterranean type environment with a strong climatic seasonality. In this zone of high agricultural activity there are artificial ponds intended for irrigation which can generate environments that constitute alternative habitats for development and nesting of waterbirds. Despite the existence of census data in these types of ponds, there are no studies addressing climatic seasonality as a factor which could influence temporal variability and abundance of the ornithological population. With this purpose, we studied temporal changes of the bird community in two types of ponds in the metropolitan region $\left(33^{\circ} 29^{\prime} \mathrm{S}-70^{\circ} 54^{\prime} \mathrm{W}\right)$ : a seasonal-type pond with pluvial regime that dries during summer season, and a managed pond, with permanent wastewater flow. In the pond with pluvial regime we observed a seasonal variation in the abundance of birds, which correlates positively with the Martonne's aridity index. On the other hand, the abundance remained relatively constant in the controlled environment, registering a non significant increase of the abundance during the summer season. Moreover, we observed in the two types of ponds; seasonal changes in the composition of the assemblage, numeric variation of certain populations and dispersion of birds between near ponds by the effects of summer desiccation. Finally, we discuss the importance of the controlled regime pond (e.g. water treatment plants or wastewater pond) as optional summer habitats during the desiccation process of seasonal sites.
\end{abstract}

KEYWORDS: Waterbirds, Martonne's aridity index, seasonality, wetlands, ponds. 


\section{INTRODUCCIÓN}

Los humedales albergan un alto número de aves residentes y migratorias, debido a su oferta de hábitats y alimento (Whittaker \& Likens 1973; Gibbs 1993; Paracuellos \& Tellería 2004). En estos ambientes, las aves acuáticas pueden ser afectadas por la disponibilidad de alimento (Marques \& Vicente 1999), el tamaño del humedal (Paracuellos \& Tellería 2004; González-Gajardo et al. 2009), salinidad, características fisicoquímicas, fluctuación del agua, profundidad y conectividad entre humedales (Fletcher \& Koford 2004; Jaksic 2004; Ma et al. 2010; Sonal et al. 2010) y por efectos antrópicos (Kusch et al. 2008). Debido a estos motivos el ensamble de aves que ocupa un hábitat determinado puede cambiar estacionalmente (Rotenberry et al. 1979; Avery \& Van Riper III 1989).

La zona central de Chile posee un clima mediterráneo, con vegetación en general formada por bosques, matorrales esclerófilos y estepas de Acacia caven (Molina) (Etienne \& Contreras 1981). El clima mediterráneo se caracteriza por ser de la zona extratropical, con precipitaciones concentradas en el período frío del año y con sequía durante la estación más cálida (Emberger 1955). De acuerdo a esto, el régimen mediterráneo no sólo implica dominancia invernal de la lluvia, sino presupone básicamente la existencia de sequía estival (Di Castri \& Hajek 1976). Actualmente en esta zona se pueden encontrar numerosos tranques artificiales de regadío al interior de predios agrícolas, los que forman sitios anegados de baja profundidad. En algunos de estos tranques artificiales (o similares), se han obtenido datos de censo de aves acuáticas (e.g. Aguirre \& Koksch 1990; Aguirre 1994; Escobar 1991; Aguirre et al. 2007) y antecedentes sobre su nidificación (Drouilli \& Ibarra 1978; Aguirre 1994; Egli \& Aguirre 1995). En base a estos antecedentes se ha concluido que estos sitios atraen un importante número de individuos, pero no poseen gran riqueza ornitológica (Schlatter \& Sielfeld en Vila et al. 2006). Pese a esto, podrían ocurrir cambios temporales en la abundancia y composición de especies en estos ambientes debido a la existencia de fluctuaciones periódicas de temperatura y pluviosidad que son propias de esta zona. Por lo tanto, el propósito de este trabajo fue (1) determinar la existencia de variación temporal en la composición y abundancia de la avifauna acuática en dos humedales de la Región Metropolitana, y (2) si estos cambios están relacionados con la estacionalidad climática.

\section{MATERIALES Y MÉTODOS}

Durante 2010 se caracterizó y cuantificó mensualmente la avifauna acuática presente en dos humedales artificiales, ubicados en la zona de Quebrada de la Plata (Región metropolitana) al interior de la Estación Agronómica
Experimental Germán Greve Silva, de la Universidad de Chile ( $\left.33^{\circ} 29^{\prime} \mathrm{S}-70^{\circ} 54^{\prime} \mathrm{W}\right)$.

En el área de estudio destaca la vegetación del tipo matorral espinoso y plantaciones frutales, además de la presencia del río Mapocho por el oeste y de un relleno sanitario a aproximadamente $3 \mathrm{~km}$ hacia el suroeste (Fig. 1).



FIGURA 1. Sitio de estudio. En el esquema superior se muestra a gran escala la localidad en que se encuentran los tranque estudiados. La imagen inferior muestra los dos tranques artificiales en donde se realizaron los censos de aves acuáticas. Abreviaciones: $\mathrm{C}=$ Cultivos, $\mathrm{ME}=$ Matorral espinoso, $\mathrm{ZU}=$ Zona urbana, $\mathrm{RM}=$ Río Mapocho, $\mathrm{RS}=$ Relleno sanitario, $\mathrm{TRP}=$ Tranque de régimen pluvial, TRC $=$ Tranque de régimen controlado.

FIgURE 1. Study site. The figure above shows at a large scale the locality in which the ponds are located. The lower image shows the two artificial ponds in which the waterbirds were censused. Abbreviations: $\mathrm{C}=$ Agricultural land, $\mathrm{ME}=$ Acacia Chaparral, $\mathrm{ZU}$ $=$ Urban zone, $\mathrm{RM}=$ Mapocho river, $\mathrm{RS}=$ Swich dam, $\mathrm{TRP}=$ Pond of rainy regime, $\mathrm{TRC}=$ Pond with permanent regime of water. 
El clima en esta zona es de tipo mediterráneo semiárido (Di Castri \& Hajek 1976), caracterizado por un periodo seco de ocho meses y uno lluvioso que comprende desde junio a septiembre (Santibáñez et al. 1990).

Uno de los humedales estudiados es un tranque artificial de regadío agrícola (llamado "El rodeo"), cuya agua proviene principalmente de la acumulación de lluvias y marginalmente se alimenta de aguas provenientes de napas subterráneas por medio de una fisura en una cisterna de almacenamiento ubicada hacia el oeste del tranque. Su superficie varía durante el año, fluctuando entre $79.000 \mathrm{~m}^{2}$ en abril y 7.500 $\mathrm{m}^{2}$ en diciembre (Solís et al. 2004). Durante el periodo seco del año, ocurre una disminución de la profundidad y superficie del espejo de agua, acompañada de la desecación total de la vegetación herbácea adyacente. El segundo humedal está ubicado a aproximadamente 283 metros hacia el sureste del primero, y también es un tranque artificial de regadío (llamado "El caimán I"). A diferencia del tranque de régimen pluvial, este último posee un régimen controlado de aguas servidas el que mantiene constante el espejo de agua durante todo el año. En este tranque, toda la parte sur se encuentra bordeada por una hilera de sauces y álamos, y la parte suroeste forma un desagüe con abundante vegetación acuática (Typha sp.).

\section{Censo de AVES}

En ambos humedales se realizó un monitoreo mensual de las aves acuáticas, según la clasificación de Victoriano et al. (2006). Según esta clasificación se consideran aves acuáticas íntegramente los órdenes Podicipediformes, Pelecaniformes, Anseriformes, Gruiformes, Charadriiformes y Suliformes, además de algunas familias de los órdenes Passeriformes y Falconiformes. Las observaciones se realizaron en los momentos de mayor actividad de las aves, entre las 08:30 a 12:00 horas, siguiendo las recomendaciones de Bibby et al. 1993. Se registraron las especies presentes y la abundancia de cada una de ellas. Por razones de accesibilidad a los sitios, durante cada conteo se muestreó primero el tranque de aguas servidas y luego el de régimen pluvial. Las observaciones y recuentos se hicieron empleando un telescopio Bausch \& Lomb (15-60 X) y binoculares Bushnell (7 x 35mm). Para identificar las especies de aves acuáticas se utilizaron las guías de campo de Jaramillo 2003 y de Araya \& Millie 2005. Durante ningún conteo se observaron desplazamientos de las aves debido a nuestra presencia.

En ambos sitios, primero se realizaron conteos independientes por tres observadores situados en un punto fijo desde altura, abarcando todo el espejo de agua. Posteriormente los valores de abundancia específica fueron promediados para cada una de las especies presentes. Luego se realizaron observaciones por la periferia de cada tranque (Bibby et al. 1993), con el objeto de corroborar la identidad de las aves presentes y fotografiarlas. Adicionalmente, para determinar el uso que hacen estas aves del ambiente, se observó paralelamente las actividades desarrolladas por las aves, realizando observaciones a distintas horas del día. En orden creciente de tiempo relativo de permanencia en estos hábitats, se definieron tres tipos de uso del ambiente: I) Uso como sitio de descanso o resguardo nocturno, entendiéndose como la dinámica de especies que sólo emplearon este ambiente en periodos definidos y breves del día o para pernoctar. II) Uso como sitio opcional de resguardo y forrajeo, se refiere a la dinámica que consistió en ocupar facultativamente este tipo de ambientes para alimentarse. III) Uso como sitio de residencia permanente, se refiere a la presencia continua de los individuos en el ambiente durante el día y la noche.

Además, se observaron indicadores de actividad reproductiva en algunas especies: cortejo, apareamiento, presencia de nidos (con o sin huevos), incubación y la presencia de polluelos y/o volantones.

\section{ANÁLISIS DE DATOS}

A NIVEL DE ENSAMBLes

Se determinó la abundancia específica (número de individuos), la riqueza (número de especies) y el índice de diversidad de Shannon (Juri \& Chani 2009; Moreno 2001) para cada mes de censo y por separado para los dos tranques.

Previo a los análisis estadísticos, se evaluó la normalidad (test de Shapiro-Wilk) y homocedasticidad (test de Bartlett) de los datos. Para los valores de abundancia en el tranque de régimen controlado, se realizó la transformación $1 /(\mathrm{x}+1)$ para cumplir con los supuestos de normalidad $\mathrm{y}$ homocedasticidad.

Para determinar si hubo diferencias en la riqueza, abundancia y diversidad dentro de cada humedal entre las estaciones $($ verano $=$ diciembre a febrero; otoño $=$ marzo a mayo; invierno $=$ junio a agosto y primavera $=$ septiembre a noviembre), se aplicó ANOVA de una vía y test de Tukey. Posteriormente, se realizó el mismo tratamiento de datos, comparando los periodos lluvioso (junio a septiembre) y seco (el resto del año).

Para examinar la similitud de la abundancia de aves entre diferentes meses, se realizó un análisis de conglomerados mediante el método UPGMA sobre el índice de similitud de Bray- Curtis (Kusch et al. 2008).

Adicionalmente, se examinó la composición estacional de la avifauna por medio del mismo método multivariado, 
pero sobre el índice de similitud de Jaccard (Arizaga et al. 2009).

Con el objetivo de explorar una posible asociación entre alguna variable comunitaria y una variable climática, se realizaron seis análisis de correlación. Las variables comunitarias fueron las descritas a comienzos de esta sección (tres por cada ambiente), y la variable climática correspondió al índice de aridez mensual de Martonne. Se empleó este índice ya que permite caracterizar temporalmente cada ambiente, interrelacionando la temperatura y las precipitaciones. Los valores de este índice aumentan a medida que las precipitaciones son más abundantes y disminuye la temperatura (Di Castri \& Hajek 1976). Este índice de aridez fue calculado a partir de datos de temperaturas medias mensuales y promedio de precipitaciones, obtenidas en la estación de Quinta Normal, ubicada a 14, $8 \mathrm{~km}$ del sitio de estudio (http://www.atmosfera.cl./datos/datos_02.html).

\section{A NIVEL DE POBLACIONES}

Se empleó el test Chi-cuadrado $\left(\chi^{2}\right)$, para evaluar si la distribución de especies de residencia permanente fue uniforme a lo largo de los meses de censo (García \& Gómez 2007). Luego, a través de la remoción secuencial de los valores de la sumatoria (relacionados con un mes) que más aportaban al valor de $\chi^{2}$, se determinaron periodos entre los cuales hubo una distribución uniforme de cada población $(\mathrm{p}>0,05)$. Para el tranque de régimen pluvial se analizaron las especies: Anas georgica (Gmelin, 1789), Himantopus melanurus (Viellot, 1817) y Fulica armillata (Viellot, 1817). Y para el ambiente de régimen controlado: Fulica rufifrons (Philippi y Landbeck, 1789) y Anas flavirostris (Viellot,
1816). En este análisis sólo se consideró a las especies relativamente más abundantes y presentes durante la mayor parte del año, ya que el uso de especies que se ausentaran algunos meses, habría generado un sesgo en los resultados. Se emplearon los programas de análisis estadístico Statistica 6.0 ( StatSoft) y Past 2.15 (Hammer 2001).

\section{RESULTADOS}

En la tabla 1, se muestran los datos censales de cada tranque, indicando las especies observadas y su abundancia mensual.

\section{A NIVEL DE ENSAMBLES}

En el tranque de régimen pluvial, la comparación de la abundancia indicó una diferencia significativa entre estaciones (ANOVA: $\mathrm{F}_{3,8}=4,12 ; \mathrm{p}=0,049$ ), sin embargo el test post hoc de Tukey no indicó diferencias $(\mathrm{p}>0,05)$. Los valores de riqueza y diversidad no presentaron diferencias estacionales (ANOVA: $\mathrm{F}_{3,8}=1,44 ; \mathrm{p}=0,301 ; \mathrm{F}_{3,8}=2,36$; $\mathrm{p}=0,147$, respectivamente). En el tranque de régimen controlado la abundancia, riqueza y diversidad no presentaron diferencias estacionales (ANOVA: $\mathrm{F}_{3,8}=0,71 ; \mathrm{p}=0,572 ; \mathrm{F}_{3,8}$ $=3,42 ; p=0,073$ y $F_{3,8}=3,09 ; p=0,09$, respectivamente). No obstante, se encontró que en el tranque de régimen pluvial la abundancia total de individuos fue significativamente mayor durante el periodo de lluvias $\left(\mathrm{F}_{1,10}=13,8 ; \mathrm{p}=0,004\right)$. Esta variación del número de aves entre periodos seco/lluvioso no fue hallada en el tranque de régimen controlado (ANOVA: $\mathrm{F}_{1,10}=0,90 ; \mathrm{P}=0,365 ;$ Fig. 2 ).



FIGURA 2. Comparación de la abundancia total de aves acuáticas entre periodos seco y lluvioso, de forma independiente en dos tranques de regadío. Las barras indican desviación estándar y las letras diferentes indican diferencias significativas $(\alpha=0,05)$. Abreviaciones: TRP $=$ Tranque de régimen pluvial; $\mathrm{TRC}=$ Tranque de régimen controlado.

FiguRE 2. Comparison of the total abundance of waterbirds between dry and rainy periods in both ponds. The bars indicate the standard deviations and the letters indicate significative differences $(\alpha=0,05)$. Abbreviations: TRP $=$ Pond with rainy regime; TRC $=$ Pond with permanent regime of water. 





En el sitio de régimen controlado, el análisis exploratorio en base al índice de similitud de Bray-Curtis (Fig. 3), reveló en términos de la abundancia, dos conglomerados, uno que contempla los meses desde octubre a enero y otro desde febrero a septiembre (índice de similitud $=0,55$ )

En el tranque de régimen pluvial, se determinó que en primavera existió una composición de especies diferente al resto del año y que la mayor similitud se obtuvo entre verano e invierno. En el ambiente de régimen controlado, la composición de especies de otoño fue diferente a la del resto del periodo y la mayor similitud se observó entre invierno y primavera (Fig. 4).

Sólo un índice comunitario presentó asociación con el índice de aridez mensual de Martonne (Fig. 5). Esta variable correspondió a la abundancia del tranque de régimen pluvial, presentando una correlación positiva y significativa $\left(\mathrm{t}_{10}=\right.$ $2,97 ; \mathrm{p}=0,014 ; \mathrm{r}=0,685)$.

\section{A NIVEL DE POBLACIONES}

Tanto en el sistema de régimen pluvial así como en el controlado, las especies analizadas presentaron una distribución no uniforme a lo largo del año $(\mathrm{p}<0,001)$. A pesar de eso, los resultados muestran que estas aves presentaron al menos durante un par de meses consecutivos una distribución uniforme de su abundancia (octubre y

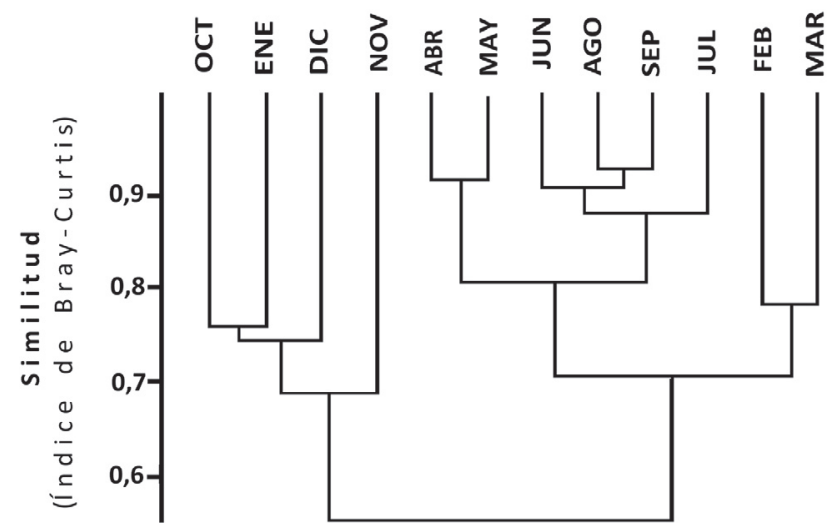

FIGURA 3. Análisis exploratorio de abundancia mensual de aves en un tranque artificial de aguas servidas. El dendrograma generado por análisis de conglomerados, muestra las similitudes de abundancia total de aves acuáticas (índice de similitud de BrayCurtis) para los meses del año 2010, en un tranque agrícola de aguas servidas.

FIGURE 3. Exploratory analysis of the monthly abundance of birds in an artificial swish pond. The dendogram generated by conglomerate analysis, shows the similarities of the total abundances of waterbirds (Similarity index of Bray-Curtis) for the months of the year 2010, in an agricultural pond composed of sewage water. noviembre). En el tranque de régimen pluvial las especies A. geórgica, H. melanurus y $F$. armillata coincidieron en la distribución uniforme durante los meses de octubre y noviembre, y en el ambiente de régimen controlado las especies F. rufifrons y A. flavirostris coincidieron durante agosto, septiembre y octubre. Los resultados de este análisis se presentan en la Tabla 2.



Figura 4. Similitud de la composición estacional de especies en dos tranques de regadío. Dendrogramas generados por análisis jerárquico de conglomerados, sobre el índice de similitud de Jaccard. En ellos, se determinó la similitud entre estaciones en términos de la presencia/ausencia de especies. Abreviaciones: $\mathrm{TRP}=$ Tranque de régimen pluvial; $\mathrm{TRC}=$ Tranque de régimen controlado.

FIgURE 4. Similarity of the seasonal composition of aquatic bird species in two irrigation ponds. The dendrogram generated by hierarchical cluster analysis over the similarity Jaccard index. The similarity among seasons in term of the presence and absence of species was performed. Abbreviations: TRP $=$ Pond with rainy regime; $\mathrm{TRC}=$ Pond with permanent regime of water.

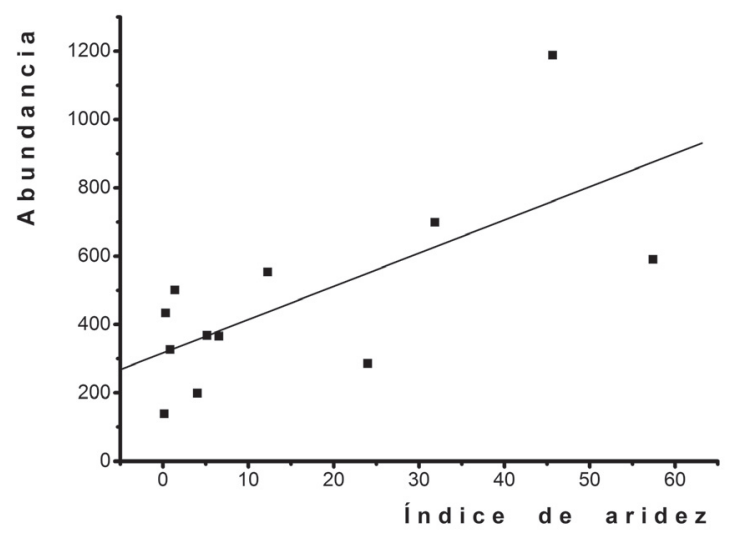

Figura 5. Análisis de correlación entre el índice de aridez de Martonne y la abundancia total mensual de aves acuáticas, en el tranque agrícola de régimen pluvial $\left(\mathrm{t}_{10}=2,969 ; \mathrm{p}=0,014 ; \mathrm{r}=\right.$ 0,685).

FIgURE 5. Correlation analysis between the Martonne's aridity index and the monthly total abundance of waterbirds, in the pond of rainy regime $\left(\mathrm{t}_{10}=2,969 ; \mathrm{p}=0,014 ; \mathrm{r}=0,685\right)$. 
La desecación del tranque de régimen pluvial, durante la transición primavera-verano, se desarrolló paralelamente a los procesos de reproducción de las especies acuáticas, generando daños no cuantificados en los nidos de taguas, los cuales perdieron la protección del agua circundante y muchos de ellos fueron depredados (Peña-Villalobos, datos sin publicar).

OBSERVACIÓN DE LA OCUPACIÓN DEL AMBIENTE Y DINÁMICAS DIARIAS,

En el tranque de régimen pluvial, se observaron los tres tipos de dinámicas diarias definidos previamente. I) Uso como sitio de descanso o resguardo nocturno: fue el tipo de dinámica observada en las especies de gaviotas Larus dominicanus (Lichtenstein, 1823) y Chroicocephalus maculipennis (Lichtenstein, 1823) que emplearon la rivera para pernoctar y descansar durante breves periodos del día. II) Uso como sitio opcional de resguardo y forrajeo: correspondió al tipo de ocupación de las especies anseriformes, las cuales ocuparon facultativamente este tipo de ambientes, siendo posible, ante la perturbación causada por un agente externo (e.g. jaurías, ganado, etc.) la formación de bandadas de decenas a centenares de individuos que se desplazaban a otros ambientes acuáticos. III) Uso como sitio de residencia permanente: fue el tipo de dinámica de la tagua $(F$. armillata), cuya ocupación de un único tranque se refleja en la respuesta a una perturbación externa, la cual consiste en agruparse al centro del espejo de agua o sumergirse en él. Estas dinámicas descritas no variaron estacionalmente.

Las especies de la familia Laridae sólo estuvieron presentes en el tranque de régimen pluvial, aun cuando estas aves no forrajeaban en él. Se observó que entre junio y octubre se produjo una disminución total del número de individuos de gaviota dominicana ( $L$. dominicanus) cerca del término de cada jornada de censo, y el retorno crepuscular de estas aves al humedal.

TABLA 2. Resultados test de Chi cuadrado. Los meses en que las especies de aves acuáticas indicadas se distribuyeron uniformemente se indican con una $\mathrm{X} . \mathrm{TRP}=$ Tranque de régimen pluvial, $\mathrm{TRC}=$ Tranque de régimen controlado.

TABLE 2. Chi square test results. X corresponds to the months in which the distribution of indicated waterbirds species was uniform. TRP $=$ Pond of rainy regime, $\mathrm{TRC}=$ Pond with permanent regime of water.

\begin{tabular}{|c|c|c|c|c|c|c|c|c|c|c|c|c|c|c|c|c|}
\hline  & Especie & $\ddot{\circlearrowright}$ & ऐ & 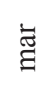 & 䒕 & 冚 & 寻 & $\Xi$ & $\stackrel{8}{\&}$ & ष्ठ & $\overleftrightarrow{0}$ & $\stackrel{?}{g}$ & $\because$ & $X^{2}$ & Df & P-value \\
\hline \multirow{3}{*}{ 急 } & A. georgica & & & & & $\mathrm{X}$ & & $X$ & & & $\mathrm{X}$ & $\mathrm{X}$ & & 6,210 & 3 & 0,102 \\
\hline & H. melanurus & & & $\mathrm{X}$ & & & & & & & $\mathrm{X}$ & $\mathrm{X}$ & & 0,506 & 2 & 0,777 \\
\hline & F. armillata & & & $\mathrm{X}$ & & & & $\mathrm{X}$ & $\mathrm{X}$ & $\mathrm{X}$ & $\mathrm{X}$ & $\mathrm{X}$ & & 5,206 & 5 & 0,391 \\
\hline \multirow{2}{*}{ 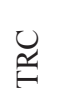 } & F. rufifrons & & $\mathrm{X}$ & & & & $\mathrm{X}$ & $\mathrm{X}$ & $\mathrm{X}$ & $\mathrm{X}$ & $\mathrm{X}$ & & & 6,87 & 5 & 0,230 \\
\hline & A. flavirostris & & & & $\mathrm{X}$ & $\mathrm{X}$ & $\mathrm{X}$ & & $\mathrm{X}$ & $\mathrm{X}$ & $\mathrm{X}$ & $\mathrm{X}$ & & 9,499 & 6 & 0,147 \\
\hline
\end{tabular}

\section{DISCUSIÓN}

\section{ESTACIONALIDAD}

En base a los análisis realizados, sólo en el ambiente de régimen pluvial se determinó una variación significativa de la abundancia entre los periodos seco y lluvioso, con un mayor número de aves durante el periodo de lluvias. Por otra parte, no se observaron diferencias significativas en la abundancia total de aves en el tranque de régimen controlado con aguas servidas. Sin embargo, se observa en él una tendencia en el incremento del promedio de la abundancia durante el periodo seco. Esta tendencia encontrada sólo en el ambiente de régimen controlado, es respaldada por estudios realizados en otros tranques artificiales de aguas servidas (Egli \& Aguirre 1995; Aguirre et al. 2007), que muestran aumentos en la abundancia y densidad de aves acuáticas durante los meses de verano (diciembre a marzo) y la disminución de estas variables entre junio y octubre (periodo lluvioso). Esto último es contrario a lo observado en el sitio de régimen pluvial en este trabajo. Este aumento estival del número de aves en sitios donde el agua posee niveles relativamente constantes, coincide temporalmente con la disminución de la abundancia total desde sitios que sufren desecación en zonas mediterráneas (Sebastián-González et al. 2009). Estos resultados sugieren que en el tranque de régimen pluvial el incremento de la temperatura promedio y la disminución 
de las precipitaciones, provocarían una reducción en la disponibilidad y/o calidad de sus recursos junto con la disminución de la superficie del agua, produciendo el desplazamiento de individuos hacia otros humedales (Ma et al. 2004) con menor variación de su régimen, siendo consistente con la predictibilidad de la abundancia en función de la superficie del humedal (Paracuellos \& Tellería 2004; González-Gajardo et al. 2009).

En el humedal de régimen pluvial, las variaciones del paisaje en función de las precipitaciones y la temperatura podrían influir en la disponibilidad de alimento de aves herbívoras, generando la disminución estival de la abundancia, en particular de patos y taguas (Lake 2011). Del mismo modo, se ha registrado en otras latitudes que las modificaciones de humedales artificiales en agroambientes originados por prolongados periodos secos, subsecuentemente generan una disminución en el número de aves, las cuales están forzadas a emplear ambientes alternativos (Sebastián-González et al. 2010). Sin embargo, la existencia de periodos de sequía y la fluctuación del nivel del agua puede afectar diferencialmente a las especies de aves. Es así como se ha reportado que especies de Ciconiiformes se ven favorecidas por la variación en el nivel del agua al proveer más oportunidades de forrajeo (Dimalexis \& Pyrovetsi 1997).

En el tranque de régimen pluvial, a pesar de no observarse cambios significativos en la diversidad ni riqueza, se evidenciaron variaciones estacionales en el ensamble de la avifauna, teniendo los meses de primavera la menor similitud con el resto del año. Estas diferencias se produjeron debido a la presencia uniforme de especies en periodo reproductivo durante octubre y noviembre, y a la aparición ocasional de especies no antes observadas, tales como: Heteronetta atricapilla (Merrem, 1841), Tringa flavipes (Gmelin, 1789) y Plegadis chihi (Viellot, 1871).

En el tranque de régimen controlado, a través de un análisis exploratorio, se observó una dicotomía entre los meses del año en términos de la abundancia, generando un conglomerado de meses formado por "octubre, noviembre, diciembre y enero" de mayor abundancia, y otro constituido por el resto del año. El aumento del número de aves, coincidiría con el periodo reproductivo de búsqueda de sitios de nidificación y refugio de algunas aves cuyos humedales de origen sufren desecación al igual que el estudiado de régimen pluvial. Específicamente, se registró un máximo en la abundancia de pato jergón grande (A. geórgica) entre octubre y noviembre. Se ha documentado recientemente que esta conectividad entre humedales artificiales puede tener diversas consecuencias en las comunidades reproductivas (Sebastián-González et al. 2010; Ma et al. 2010). En términos de la composición de la comunidad de aves, se observó por medio de otro análisis exploratorio (similitud de
Jaccard) diferencias entre otoño y el resto de las estaciones. Esta diferencia se generó dada la baja riqueza ornitológica y la alta variación mensual de la composición de especies, características que podrían estar relacionadas con el carácter transitorio que posee esta estación entre el periodo seco y lluvioso, y no se asociaría en este caso con un influjo de aves migratorias como se ha documentado para humedales costeros (Simeone et al. 2008). Parámetros tales como la riqueza y la diversidad no presentaron variación en ninguno de los dos ambientes, lo que coincide con otros estudios en agroambientes de España (Sebastián-González et al. 2010).

\section{Poblaciones}

En relación a la frecuencia de observación de aves acuáticas en los dos tranques estudiados, se identificaron especies ocasionales y otras residentes, las que en su mayoría emplearon estos sitios para la nidificación entorno a los meses de octubre y noviembre, excepto por las aves de las familias Laridae, Ardeidae y Podicipedidae.

Se determinó que las poblaciones con actividad reproductiva (A. geórgica, H. melanurus, F. armillata, F. rufifrons y A. flavirostris) mantuvieron una distribución uniforme a través de los meses de nidificación.

En el ambiente de régimen controlado se identificó que las especies pato jergón chico (A. flavirostris) y tagua ( $F$. rufifrons) presentaron una distribución uniforme de su abundancia en el tiempo, que en promedio abarcó más meses que en las especies del sitio de régimen pluvial. Esta menor variación de la abundancia mensual de las poblaciones, podría estar relacionada con la estabilidad anual del nivel del agua y otros factores afectados a consecuencia de esto, tales como la existencia de sitios adecuados para la nidificación y cría (Ma et al. 2010).

En el tranque de régimen pluvial, se determinó que la población de taguas presentó la menor variación mensual y que la población de pato jergón grande mantuvo una abundancia uniforme durante los cuatro meses de primavera. Esto podría deberse a que esta especie junto con otros anseriformes poseen una ocupación del ambiente opcional frente a otros sitios de similares características, asociándose su presencia positivamente al tamaño del humedal (Sebastián-González et al. 2010) y prefiriendo tranques con niveles estables de agua (Gordon et al. 1998). Además, otras investigaciones sugieren que los humedales con niveles constantes de agua son más convenientes para la reproducción de Gruiformes y Anseriformes (Krapu 1970; Laperle 1974).

Estudios que abordan la asociación entre la presencia de aves acuáticas y las características que los agroambientes en que se encuentran (Sebastián-González et al. 2010) 
sugieren en el caso de las gaviotas (Larus sp.), una predilección por grandes estanques durante invierno. Estas aves, posiblemente se movilizaban hacia el relleno sanitario presente en las cercanías del sitio de estudio. Esta suposición estaría fuertemente respaldada por la dirección de vuelo de estas aves en dirección suroeste y por la coincidencia de esta actividad observada con las fluctuaciones diarias de actividad reportadas para el mismo relleno sanitario, luego de tres años de observación (Lobos et al. 2011).

Finalmente, los humedales artificiales de régimen controlado, permitirían albergar numerosas especies de aves acuáticas nativas durante procesos de desecación estival de causes naturales y tranques estacionales. De este modo, su presencia favorecería la nidificación y reproducción de ciertas especies en zonas mediterráneas, principalmente Anseriformes y Gruiformes, generando un impulso contrario a la disminución de la diversidad producto de la destrucción de humedales. En este sentido, para la comprensión íntegra de los procesos de interconexión de humedales o tranques, se propone la realización de estudios que aborden la dinámica espacial de las aves acuáticas en aguas continentales y la identificación de los factores bióticos y abióticos que originarían el desplazamiento y preferencia por ciertos humedales artificiales.

\section{AGRADECIMIENTOS}

Al Administrador de la Estación Agronómica Germán Greve Silva, Marcelo Orellana, por su hospitalidad, facilitar acceso a los humedales e instalaciones. A Flavia González Díaz, por su apoyo en terreno y a los dos revisores anónimos por sus valiosas sugerencias.

\section{BIBLIOGRAFÍA}

AguirRe, J. 1994. Nidificación de aves acuáticas en el fundo San Rafael. Boletín chileno de ornitología 1: 2-7.

Aguirre, J. \& KoKsch, H. 1990. Diversidad y censo anual de la comunidad de aves de las lagunas de Llo- 1leo, comuna de San Antonio, V Región (33 36'S y $\left.71^{\circ} 38^{\prime} \mathrm{W}\right)$. Boletín informativo Unorch, 10: 16-17.

Aguirre, J., Rubio, M. \& CAnepa, A. 2007. Variación interanual de la avifauna acuática presente en la planta de tratamiento de aguas servidas Santiago poniente. Boletín Chileno de Ornitología 13: 2-1.

Araya, B. \& Millie, G. 2005. Guía de campo de las aves de Chile. Editorial Universitaria, IX Edición.

Arizaga, J., Alonso, D., Fernández, E., Fernández, I., Martín, D. \& Vilches, A. 2009. Ensamblaje de aves passeriformes y su dinámica a lo largo del ciclo anual en un carrizal del valle del Ebro. Ecología 22: 101-116.

Avery, M.L. \& VAN RIPER III, C. 1989. Seasonal changes in bird communities of the Chaparral and Blue-oak woodlands in central California. The Condor 91: 285-295.

Bibby, C., Burgess, N. \& Hill, D. 1993. Birds census techniques. Academic press limited.

Connor, K. \& GaBor, S. 2006. Breeding waterbird wetland habitat availability and response to water-level management in Saint John River floodplain wetlands, New Brunswick. Hydrobiologia. 567: 169-181.

Di Castri, F., \& Hajek, E. 1976. Bioclimatología de Chile. Bicerrectoría Académica Universidad Católica de Chile.

Dimalexis, A., Pyrovetsi, M. 1997. Effect of water level fluctuations on wading bird foraging habitat use at an irrigation reservoir, Lake Kerkini, Greece. Colonial Waterbirds, 20:244-252.

Drouilli, P. \& Ibarra, J.C. 1978. Presencia y reproducción de patos (Anseriformes) en la reserva forestal Lago Peñuelas, Valparaíso, durante 1976. Boletín ornitológico, Unorch 10: 6-8.

Egli, G. \& Aguirre, J. 1995. Abundancia, Riqueza y frecuencia de ocurrencia y estado de conservación de la avifauna de ambientes acuáticos del tranque San Rafael, comuna de Lampa Región Metropolitana. Boletín chileno de ornitología, 2: 14-20.

Emberger, L. 1955. Projet d'une classification biogéographique des climats. Annee Biologique, 31(5-6): 249 -255.

Escobar, P. 1991. Tranque San Rafael: Censo enero 1991. Boletín informativo Unorch, 11: 10-12.

Etienne M. \& Contreras D. 1981. Cartografía de la vegetación y sus aplicaciones en Chile. Boletín Técnico, Universidad de Chile. 46: 1-80.

Facultad de Ciencias Físicas y Matemáticas (FCFM). 2010. Departamento de Geofísica (DGF) de la Universidad de Chile. URL: http://www.atmosfera.cl./HTML/datos/ datos_02.html Accesado: Diciembre 20, 2010.

Fletcher, R.J. \& Koford, R.R. 2004. Consequences of rainfall variation for breeding wetland blackbirds. Canadian Journal of Zoology. 82: 1316-1325.

García, G. \& Gómez, A. 2007. Abundancia y riqueza específica en un ensamble de aves marinas y costeras del sudeste de la provincia de Buenos Aires, Argentina. Hornero 22(1): 9-16.

GiBBS, J.P. 1993. The importance of small wetlands for the persistence of local populations of wetland-associated animals. Wetlands 13: 25-31.

GonzÁlez-Gajardo, A., Victoriano, P. \& Schlatter, P. Waterbird Assemblages and Habitat Characteristics in Wetlands: Influence of Temporal Variability on Species-Habitat Relationships. Waterbirds 32(2): 225-233.

Gordon, D., Gray, B. \& Kaminski, R. 1998. Dabbling duck-habitat associations during winter in coastal South Carolina. Journal of Wildlife Management 62(2): 569-580.

Hammer, Ø., Harper, D.A.T., \& P.D. Ryan. 2001. PAST: Paleontological Statistics Software Package for Education and Data Analysis. Palaeontologia Electronica 4(1): 9pp. http://palaeo-electronica.org/2001_1/past/issue1_01.htm

Jaramillo, A. 2003. Birds of Chile. Princeton Field Guides. Princeton and Oxford.

JAKSIC, F.M. 2004. El niño effects on avian ecology: lessons learned from the southeastern pacific. Ornitologia Neotropical 15 (Suppl.): 61-72.

JURI, M. \& CHANI, J. 2009. Variación estacional en la composición de las comunidades de aves en un gradiente urbano. 
Ecología Austral 19: 175-184.

Kopachena, J. \& Evans, R. 1990. Flock recruitment in Franklin's gulls. Colonial Waterbirds 13: 92-95.

Krapu, G.L., Parsons, D.R. \& Weller, M.W. 1970. Waterfowl in Relation to Land Use and water Levels on the Spring Run area. lowa State Journal of Science. 44(4) 437-452.

Kusch, A., CÁrcamo, J., \& Gómez, H. 2008. Aves acuáticas en el humedal urbano de tres puentes, Punta Arenas (53 $\mathrm{S})$, Chile austral. Anales Instituto Patagonia (Chile) 36(2): 4551.

LAKE, P. S. 2011. Drought and Aquatic Ecosystems: Effects and Responses. John Wiley \& Sons.

LAPERLE, M. 1974. Effects of water level fluctuation on duck breeding success, pp. 18-30. En: Canadian Wildlife Service Waterfowl Studies in Eastern Canada, 1969-73, pp. 106. Canadian Wildlife Service Report. Ser. 29

Lobos, G., Bobadilla, P., Alzamora, A. \& Thomson, R. 2011. Patrón de actividad y abundancia de aves en un relleno sanitario de Chile central. Revista Chilena de Historia Natural 84: 107-113.

MA, Z., Li, Bo., ZhaO, B., Jing, K., TAng, S. \& Chen, J. 2004. Are artificial wetlands good alternatives to natural wetlands for waterbirds? - A case study on Chongming Island, China. Biodiverdity and Conservation. 13: 333-350.

MA, Z., CAI, Y., LI, B. \& Che, J. 2010. Managing Wetland Habitats for Waterbirds: An International Perspective. Wetlands 30: $15-2$.

Marques, P. \& Vicente, L. 1999. Seasonal variation of vwaterbird prey abundance in the sado estuary rice field. ARDEOLA 46(2): 231-234.

Moreno, C.E. 2001. Métodos para medir la biodiversidad. M\&TManuales y Tesis SEA, vol.1.

Ogden, J.C .1994. A comparison of wading bird nesting colony dynamics (1931-1946 and 1974-1989) as in indication of ecosystem conditions in the Southern Everglades. En: Everglades: The ecosystem and its restoration (Eds. Davis SM, Ogden JC). pp 533-570.

Paracuellos, M. \& Tellería, J.L. 2004. Factors Affecting the distributionof a waterbird comunity: the role of habitat configuration and bird abundance. Waterbirds 27(4): 446453.

Rotenberry, J.T., Fitzner, R.E. \& Rickard, W.H. 1979. Seasonal variation in avian community structure: differences in mechanism regulating diversity. The Auk 96: 449-505.

SANDFord, D.S. 1980. Wildlife Management Techniques Manual. The Wildlife Society. 375 pp.

Santibañez, F., Uribe, J. \& Vicencio, M. 1990. Atlas Agroclimático de Chile: Regiones V y Metropolitana. Universidad de Chile, Facultad de Ciencias Agrarias y Forestales.

Sebastián-González, E., Sánchez-Zapata, J. \& Botella, F. 2010. Agricultural ponds as alternative habitat for waterbirds: spatial and temporal patterns of abundance and management strategies. European Journal of Wildlife Research 56: 11-20.

Simeone, A., Oviedo, E., Bernal, M. \& Flores, M. 2008. Las aves del humedal Mantagua: riqueza de especies, amenazas y necesidades de conservación. Boletín Chileno de Ornitología 14(1): 22-35.

Solís, R., Lobos, G. \& Iriarte, A. 2004. Antecedentes sobre la biología de Xenopus leavis y su introducción en Chile. Universidad de Chile / Servicio Agrícola y Ganadero, pp 61.

Sonal, D., Jargruti, R. \& Geeta, P. 2010. Avifaunal diversity and water analysis of an inland wetland. Journal of Wetlands Ecology 4: 1-32.

Victoriano, P., González, A. \& Schlatter, R. 2006. Estado de conocimiento de las aves de aguas continentales de Chile. Gayana 70(1): 140-162.

Schlatter, R. \& Sielfeld, W. 2006. Avifauna y mamíferos acuáticos de los humedales de Chile. En: Macrófitas y vertebrados de los sistemas límnicos de Chile (Eds. Vila I., Veloso A., Schlatter R. \& Ramiréz C.), pp. 169. Editorial Universitaria.

Whittaker, R.H. \& Likens, G.E. 1973. Primary production: the biosphere and man. Human Ecology 1: 357-369.

Recibido: 15.06 .11

Aceptado: 08.05.12 